\title{
Structural studies of three-arm star block copolymers exposed to extreme stretch suggests persistent polymer tube
}

Mortensen, Kell; Borger, Anine L.; Kirkensgaard, Jacob J.K.; Garvey, Christopher J.; Almdal, Kristoffer; Dorokhin, Andriy; Huang, Qian; Hassager, Ole

Published in:

Physical Review Letters

Link to article, DOI:

10.1103/PhysRevLett.120.207801

Publication date:

2018

Document Version

Publisher's PDF, also known as Version of record

Link back to DTU Orbit

Citation (APA):

Mortensen, K., Borger, A. L., Kirkensgaard, J. J. K., Garvey, C. J., Almdal, K., Dorokhin, A., Huang, Q., \& Hassager, O. (2018). Structural studies of three-arm star block copolymers exposed to extreme stretch suggests persistent polymer tube. Physical Review Letters, 120, [207801].

https://doi.org/10.1103/PhysRevLett.120.207801

\section{General rights}

Copyright and moral rights for the publications made accessible in the public portal are retained by the authors and/or other copyright owners and it is a condition of accessing publications that users recognise and abide by the legal requirements associated with these rights.

- Users may download and print one copy of any publication from the public portal for the purpose of private study or research.

- You may not further distribute the material or use it for any profit-making activity or commercial gain

- You may freely distribute the URL identifying the publication in the public portal 


\title{
Structural Studies of Three-Arm Star Block Copolymers Exposed to Extreme Stretch Suggests a Persistent Polymer Tube
}

\author{
Kell Mortensen, ${ }^{*}$ Anine L. Borger, and Jacob J. K. Kirkensgaard \\ Niels Bohr Institute, University of Copenhagen, Copenhagen, Denmark \\ Christopher J. Garvey \\ Australian Nuclear Science and Technology Organisation, Lucas Heights, New South Wales 2234, Australia \\ Kristoffer Almdal and Andriy Dorokhin \\ Department of Micro- and Nanotechnology, Technical University of Denmark, 2800 Kongens Lyngby, Denmark \\ Qian Huang and Ole Hassager \\ Department of Chemical and Biochemical Engineering, Technical University of Denmark, 2800 Kongens Lyngby, Denmark
}

(Received 5 January 2018; revised manuscript received 16 March 2018; published 16 May 2018)

\begin{abstract}
We present structural small-angle neutron scattering studies of a three-armed polystyrene star polymer with short deuterated segments at the end of each arm. We show that the form factor of the three-armed star molecules in the relaxed state agrees with that of the random phase approximation of Gaussian chains. Upon exposure to large extensional flow conditions, the star polymers change conformation resulting in a highly stretched structure that mimics a fully extended three-armed tube model. All three arms are parallel to the flow, one arm being either in positive or negative stretching direction, while the two other arms are oriented parallel, right next to each other in the direction opposite to the first arm.
\end{abstract}

DOI: 10.1103/PhysRevLett.120.207801

The flow and deformation of macromolecules are ubiquitous in nature as well as industry. A microscopic understanding of such macromolecular flow behavior has accordingly significant interest. Important parameters for the flow behavior include both the molecular chemistry and the molecular architecture, but also the exact flow condition. The tube model of polymers proposed and described by de Gennes [1] and Doi and Edwards [2] has proven to make a very good theoretical basis for the observed flow characteristics of linear chains [3,4], even though some modifications have been proposed to account for details in the experimental findings [4-8]. Large strain amplitudes can be expected to show further complications beyond the properties of the linear regime described in the tube model $[9,10]$.

It has been questioned whether the tube model can describe branched and other types of complex polymer systems [11]. Star polymers, for example, have been the subject of several studies in both melts and solutions [11-20]. Dondos et al. discussed the elongation of starshaped polystyrenes in flowing solutions, showing how the molecular deformation depends on their number of branches [13]. Ripoll et al. made mesoscale simulations showing that star polymers in shear flow change from linear-polymer-like to capsule-like behavior with increasing functionality [17]. $\mathrm{Xu}$ and Chen made a coarse grained molecular dynamic simulation, showing that star polymers align and deform for shear rates beyond a critical value [19]. Huang et al. measured the nonlinear rheology of three-arm star polymer melts in fast extensional flows [20], showing that the extensional viscosity of star polymers was identical to that of linear polymer in agreement with theoretical predictions [21]. While the agreement between the extensional viscosity and the model prediction is significant, it provides at best an indirect confirmation that branched polymers in strong extensional flows become quasilinear by aligning the arms.

In this Letter, we present experimental studies of the simplest complex polymer system that provides direct evidence from which the arm configuration in strong extensional flow may be tested. By using a deuterium labeled three-arm star, we show that the configuration corresponds very well to that of a fully extended threearmed tube. This does not necessarily imply that all dynamics of a star polymer can be associated to a tube model, and one may claim that the basis of the tube model, the entanglements, disappear when a tube becomes fully extended. However, it is striking how well the characteristics of a three-armed tube can explain our rather distinct experimental observations.

The Letter is restricted to the structure as measured in the relaxed state, and immediately after stopping the flow. The aim of the synthesis was for three-armed polystyrene star polymers labeled by hydrogen-deuterium exchange at the very end of each polymer arm, as sketched in Fig. 1, to 
3-Arm Polystyrene Star-Polymer

Each arm has end-block of deuterated monomers

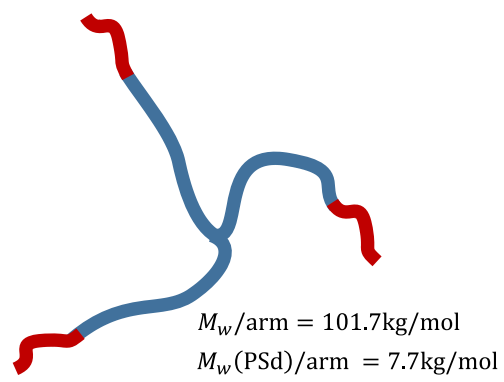

FIG. 1. Schematic illustration of the star copolymer where each arm is a polystyrene block copolymer of total $101.7 \mathrm{~kg} / \mathrm{Mol}$ molar mass, made up of a $94 \mathrm{~kg} / \mathrm{Mol}$ normal PS block near the star center, and $7.7 \mathrm{~kg} / \mathrm{Mol}$ deuterated end blocks (PSd).

obtain contrast for neutron scattering experiments. The deuterated blocks are chosen to be relatively small to get more precise and easily accessible information on the star polymer deformation, even though the small size causes rather weak scattering and thereby somewhat noisy data. The resulting molar masses of the polystyrene samples were determined using size exclusion chromatography with nonstabilized tetrahydrofuran (THF) as the eluent and applying a column set consisting of a $5 \mu \mathrm{m}$ guard column and two $300 \times 8 \mathrm{~mm}^{2}$ columns. The system was equipped with a triple detector system consisting of a combined Viscotek model 200 differential refractive index (DRI), a differential viscosity detector, and a Viscotek (LD 600) right angle laser light scattering detector (RALLS). The overall molar mass is calculated based on both the RALLS and DRI signal, whereas the polydispersity is based on calibration with polystyrene standards. The synthesis of three-arm polystyrene- $b$-polystyrene-d8 star block polymers was carried out in freshly distilled THF in three stages [22]. Synthesizing short narrow molar mass distribution polymer blocks is difficult in polar solvents due to high polymerization rates compared to the mixing rate and results in high polydispersity. The problem was minimized by performing the reactions of the labeled linear polystyrene-d8 polymer at $-108^{\circ} \mathrm{C}$, close to the freezing point of the solvent. The resulting polymers have a molar mass of $M_{w}=7.7 \mathrm{~kg} / \mathrm{mol}$ with a polydispersity of 1.14 . During the second stage the nondeuterated monomers were added while the reaction mixture was heated up to $-78^{\circ} \mathrm{C}$. The polydispersity of the full block copolymer arm is 1.40 due to incomplete homogeneity in the synthesis in this step. The resulting linear PS-PSd block copolymer has total molar mass $M_{w}$ of $101.7 \mathrm{~kg} / \mathrm{mol}$ corresponding to a mass fraction of deuterated polystyrene $f$ equal to 0.075 . The final star formation stage was made at $-78^{\circ} \mathrm{C}$ with methyltrichlorosilane as the coupling agent. The resulting polystyrene star polymer solution was fractionated and dried in vacuum at $50{ }^{\circ} \mathrm{C}$ for $24 \mathrm{~h}$, resulting in an amorphous sample of threearm block copolymer stars with molar mass equal to
$309.1 \mathrm{~kg} / \mathrm{mol}$ and polydispersity equal to 1.30 . The relative large polydispersity may reflect a small amount long linear block copolymers, which may be the consequence of nonoptimal temperature during the polymer synthesis. The quality of the polymer was investigated further using rheology comparing the polymer of the present Letter with that of a well-defined low-PDI threearm fully protonated PS star (PDI < 1.1) [20]. Rheology measurements show that the relaxation of the partly deuterated material is only slightly slower at low frequency, indicating some side product of a high-molar mass linear PS.

For polystyrene, the polymer end-to-end statistical length is $\sqrt{\left\langle R_{\circ}^{2}\right\rangle}[\AA]=\sqrt{0.437 M_{w}[\mathrm{~g} / \mathrm{mol}]}$, the Kuhn segment length is $b=18 \AA$, the entanglement molar mass is $M_{e}=$ $16.6 \mathrm{~kg} / \mathrm{mol}$ and the tube diameter is $a=85 \AA$ [23]. With the molar mass $(M=101.7 \mathrm{~kg} / \mathrm{mol})$ for each arm, the number of entanglements is $Z=101.7 / 16.6 \approx 6$, and the equilibrium end-to-end length is $R_{\circ}(1 \mathrm{arm})=$ $\sqrt{0.437 \times 101.700} \AA \approx 210 \AA$. The number of segments in each arm is $N_{\text {arm }}=\left(R_{\circ} / b\right)^{2}=(210 / 18)^{2}=135$, and thus the total number of segments in the star polymer, $N=3 N_{\text {arm }}=405$.

The samples were stretched with the VADER 1000, Rheo Filament ApS. As shown by Hengeller et al. extensional steady state flow conditions are established beyond a Hencky strain of $\epsilon=3$, which is the Hencky strain applied in the present study [24]. The applied strain rate for the stretched sample was $\dot{\epsilon}=0.06 \mathrm{~s}^{-1}$, performed at $T=125^{\circ} \mathrm{C}$. The equilibrium Rouse time of a linear chain of the same span $M_{W}$ is $450 \mathrm{~s}$ at $125^{\circ} \mathrm{C}$ [20]. The nondimensional stretch rate based on this time constant is significantly greater than unity so we expect that the molecules are highly oriented and nonlinearly stretched. The samples were quenched with a cooling rate of about $10 \mathrm{~K} / \mathrm{s}$ as described in Kirkensgaard et al. [10]. Since the glass transition temperature is $105^{\circ} \mathrm{C}$, the melt solidifies in a time much shorter than the Rouse time. Therefore, the molecular configurations in the solid samples are assumed to be identical to those in the melt at the time of quench. The large deformation does, according to previous related studies, not cause degradation of the complex polymer molecules [20].

The structural studies were performed using small-angle neutron scattering (SANS). SANS data were obtained using the SANS-1 instrument at PSI, Switzerland and Quokka at ANSTO, Australia. Results obtained by the two instruments agree perfectly. The results shown below are all obtained using the Quokka instrument, where two instrumental settings were applied, both with $5 \AA$ neutrons with $10 \%$ wavelength resolution and the collimation defined by a source diameter of $50 \mathrm{~mm}$ and a pinhole in front of the sample with a diameter of $10 \mathrm{~mm}$. One instrumental setting had $10 \mathrm{~m}$ collimation length and $5 \mathrm{~m}$ sample-to-detector 

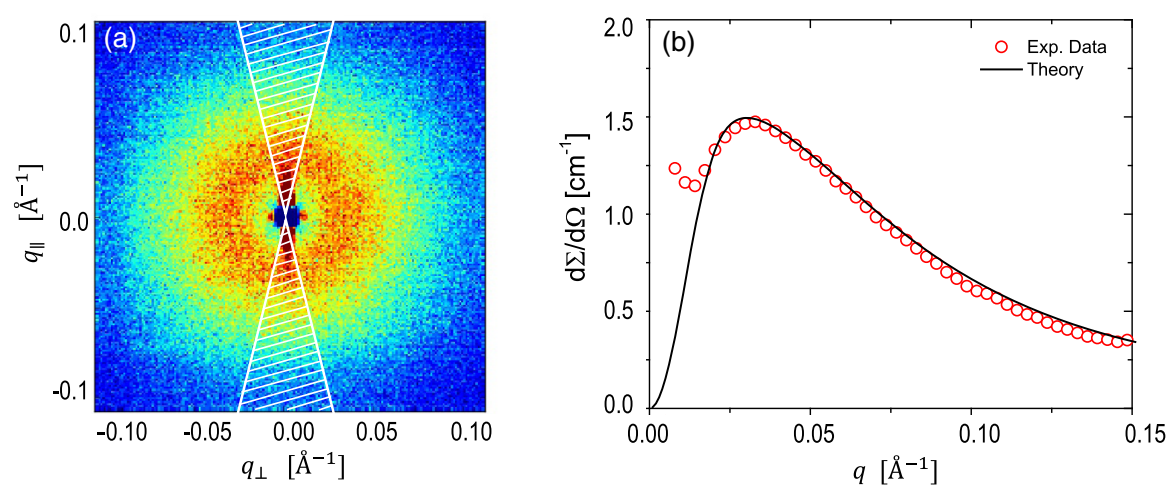

FIG. 2. Small-angle neutron scattering study of the relaxed star-polymer system. (a) The 2D scattering pattern as obtained in the SANS instrument. (b) The azimuthally averaged intensity versus scattering vector, as obtained from the nonstriped area in (a).

distance, giving a $q$ range equal to $0.01-0.15 \AA^{-1}$, while the second setting used collimation length and sample-todetector distance both equal to $14 \mathrm{~m}$, giving a $q$ range of $0.004-0.06 \AA^{-1}$.

Figure 2 shows the 2D scattering function of the fully relaxed star polymer. The rather poor statistics is due to a limited amount of material and only rather small scattering units in the labeled polymer. Unfortunately, this sample is affected by artificial small-angle scattering, which arises from the sample itself, but is likely related to cracks that we only realized after finishing the SANS measurements. The other measured samples do not have similar problems. Fortunately, the significant small-angle scattering does not disturb the main scattering characteristics of the correlation peak. The cylindrical shape of the sample gives further rise to some small angle scattering horizontally near the beamstop.

Figure 2(b) shows the azimuthally averaged structure factor $I(q)$, where $q=4 \pi / \lambda \sin (\theta)$ is the scattering vector, $\lambda$ being the neutron wavelength and $\theta$ half the scattering angle. The averaged data exclude the striped area in Fig. 2(a) to limit the effect of artificial background. The incoherent scattering corresponding to pure polystyrene was subsequently subtracted [25], to show the pure starblock copolymer form factor. The SANS scattering pattern of the relaxed sample is characterized by a broad peak centered at $q^{*}=0.03 \AA^{-1}$, corresponding to a characteristic length scale of $d=2 \pi / q^{*}$ equal to $210 \AA$.

If we assume an ideal random position of the star molecules, there will be no intermolecular correlations. The scattering pattern will accordingly be dominated by the form factor of a single star molecule, which can be calculated based on a random phase approach equivalent to the calculation of a diblock copolymer melt developed by Leibler [26]. Using such a RPA method, strictly following the calculations of M. Doi [27], the form factor can be expressed as

$$
I(q)=\frac{N}{S / W-2 \chi N}
$$

with $N$ being the total degree of polymerization, $\chi$ being the Flory-Huggins interaction parameter, and $S$ and $W$ are given by

$$
S=S_{A A}+S_{B B}+2 S_{A B}
$$

and

$$
W=S_{A A} S_{B B}-S_{A B}^{2}
$$

respectively, where the partial structure factors for a threearm star polymer are given by [28-31]

$$
\begin{aligned}
S_{A A}= & -3 h[(1-f) / 3]+3 h[2(1-f) / 3] \\
S_{B B}= & 3\{h(f / 3)+h(2 / 3)+h[2(1-f) / 3] \\
& -2 h[(2-f) / 3]\} \\
S_{A B}= & 3 / 2 h[(1-f) / 3]-3 / 2 h(1 / 3)-3 / 2 h(f / 3) \\
& +3\{h[(2-f) / 3]-h[2(1-f) / 3]\},
\end{aligned}
$$

where $h(x)$ is the generalized $q$-dependent Debye function introduced by Leibler:

$h(x)=2 /\left(q R_{g, N}\right)^{4}\left\{x\left(q R_{g, N}\right)^{2}+\exp \left(-x\left(q R_{g, N}\right)^{2}\right)-1\right\}$

where $R_{g, N}$ is the radius of gyration of a linear polymer with similar degree of polymerization $(N)$. Heinrich et al. made a corresponding analysis on end-labeled $\mathrm{H}$-shaped molecules, studying the conformational changes upon more gentle stretching [32].

While small-angle neutron scattering is typically done on an absolute scale, this is not trivial for the cylindrical samples, where transmission and thickness vary over the beam. In attempt, anyway, to give our data on an absolute scale, we presuppose that the asymptotic high- $q$ scattering is dominated by incoherent scattering from pure polystyrene [25]. The data shown in Fig. 2 have thus been 

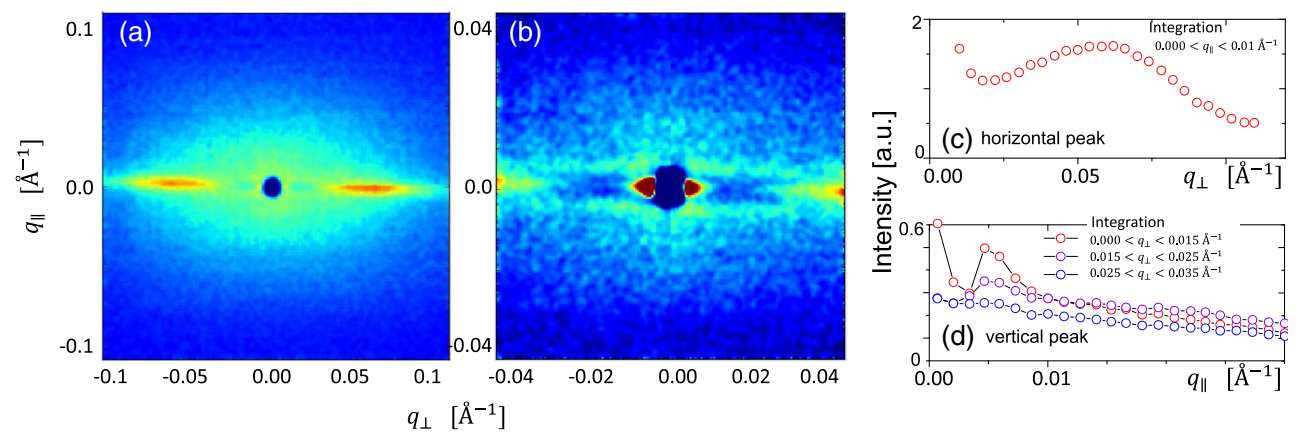

FIG. 3. Experimental small-angle neutron scattering results of a star-polymer system exposed to elongational stretching (vertically) using two instrumental settings at the Quokka instrument: neutron wavelength $\lambda=5 \AA$ and sample to detector distance equal 5 m (a) and $14 \mathrm{~m}$ (b), respectively. (c) The horizontal peak intensity versus $q_{\perp}$ as averaged over a $q_{\|}$slab. (d) The intensity of the vertical peak versus $q_{\|}$as averaged over different $q_{\perp}$ slabs.

normalized using the incoherent scattering from polystyrene. The incoherent scattering was subsequently subtracted, to show the pure star-block copolymer form factor.

The solid line in Fig. 2 shows the RPA form factor calculated according to Eqs. (1) and (2) using the known molecular parameters given above, i.e., $N=405$, $f=0.075$. The Flory-Huggins interaction parameter $\chi(\mathrm{PS}-\mathrm{PSd})=0.004$ [33]; however, the scattering function is rather insensitive to changes in $\chi$, reflecting that the system is far from any microphase separation. The theoretical scattering function is arbitrarily multiplied by 1.05 , to fit the experimental data, which absolute intensity is anyway somewhat uncertain, according to the discussion above. The experiment and the theory agree very well, but the peak position is slightly shifted relative to the theoretical expectation, which can be explained by an effective segment-number $N$ somewhat smaller than expected, or it may reflect a small fraction of two-arm stars.

The experimental 2D-SANS data, as obtained immediately after elongational flow, is shown in Fig. 3. The data show a very characteristic pattern dominated by two sets of correlation peaks: one pair at the horizontal axis around $q_{\perp}= \pm 0.06 \AA^{-1}$ [Figs. 3(a) and 3(c)] and another pair centered at the vertical axis around $q_{\|}= \pm 0.005 \AA^{-1}$ [Figs. 3(b) and 3(d)]. Both sets of correlation peaks have very large horizontal dispersions, i.e., perpendicular to the flow direction, while being highly confined vertically parallel to the flow. The characteristics are further clear from Figs. 3(c) and 3(d), showing intensity averaged over slabs parallel to, respectively, $q_{\perp}$ and $q_{\|}$, as expressed in the figures. The SANS pattern has additional horizontal scattering near the beamstop, which is attributed reflection from the thin, cylinder shaped samples. This scattering is not discussed further. The pattern is further characterized by an underlying close-to ellipsoidal contour pattern, which may reflect the form factor of the deformed deuterated chains.

To make the discussion more clear, we have combined the two data sets into one, and given a schematic illustration of the findings, as shown in Figs. 4(a) and 4(b), respectively. It is interesting to note that the two types of peaks do not merge; i.e., they are not part of a common ellipsoidal (or other simple) correlation ring as might have been expected to emerge from "deformation" of the relaxed pattern shown in Fig. 2. The peaks, on the contrary, make up four stripes running parallel in the two-dimensional detector plane, as also clear from Fig. 3(d) showing constant peak value $q_{\|}^{*}= \pm 0.005 \AA^{-1}$. This strongly indicates that the two types of structures reflect properties of uncorrelated origin.

To discuss the origin of the scattering pattern, we note that the molecules must be highly stretched, implying that the RPA model should not be used. On the contrary, we propose a most simple model with geometrically simple, stretched objects where the scattering peaks as a consequence of the Fourier transformation simply reflect distances between the labeled blocks.

The two peaks centered on the vertical $q_{\|}$axis at $q_{\|}^{*}=$ $\pm 0.005 \AA^{-1}$ have character of horizontal streaks extending to rather large $q_{\perp}$ values (beyond the $q_{\perp}^{*}$ of the "horizontal" peaks discussed below). Such characteristics may have a very simple origin. The Fourier transform, and thereby the

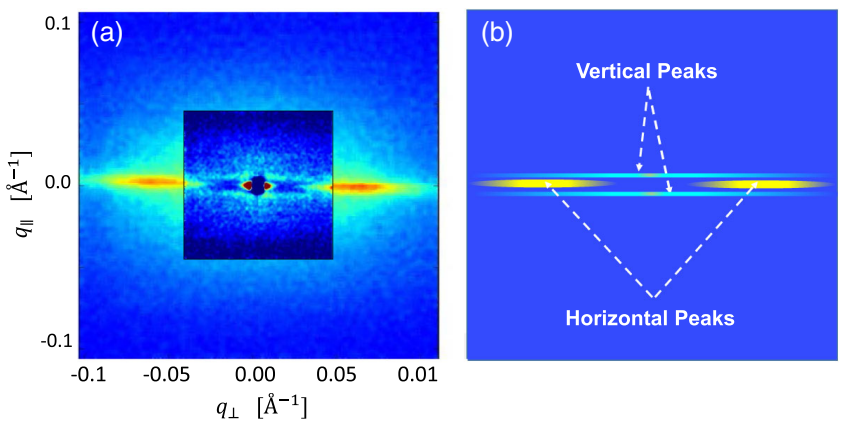

FIG. 4. Small-angle neutron scattering study of star-polymer system exposed to elongational stretching. (a) The 2D experimental scattering pattern as obtained in the SANS instrument. (b) A schematic illustration of the findings to clarify the discussion in the text. 
scattering pattern, of a simple one-dimensional model with two elongated scattering domains aligned in the flow direction will have exactly such characteristics with the peak-width being relatively confined in the vertical direction parallel to flow, but broad perpendicular to the flow determined by the width of the deuterated block. The pattern thus reflects the correlation between two $7.5 \mathrm{~kg} / \mathrm{mol}$ deuterated blocks parallel to the flow, one block in positive and one in negative stretching direction, where the center value $q_{\|}^{*}=0.005 \AA^{-1}$ corresponds to a distance of roughly $1300 \AA$.

The two peaks centered on the horizontal $q_{\perp}$ axis are also very broad parallel to $q_{\perp}$ while being very confined in the $q_{\|}$direction parallel to the flow. The center values $q_{\perp}^{*}=$ $\pm 0.06 \AA^{-1}$ correspond to a distance of the order of $100 \AA$. The origin of these peaks must be correlations between two deuterated blocks that have oriented into the same direction relative to flow. The significant confinement in the vertical direction implies that the two scattering domains are positioned in the same plane perpendicular to flow, implying that in a given star molecule, both chains are stretched by the same factor. The large horizontal width of the reflections may have two origins: it may reflect diversity in distance between the domains and it may reflect the form factor of the stretched deuterated blocks. Some variation in the distance is reasonable, and the stretched chain may very well be relatively thin, as also argued for the horizontal width of the other pair of peaks.

To understand these findings on the molecular level, it is relevant to look into the characteristic polystyrene dimensions given above. The horizontal peaks are attributed to correlation between the deuterated domains of two arms that are stretched into the same direction relative to the flow (Fig. 5). The distance of roughly $100 \AA$ resulting from the horizontal peaks is, with the given statistics, very close to the tube diameter $a=85 \AA$ and thereby the tube

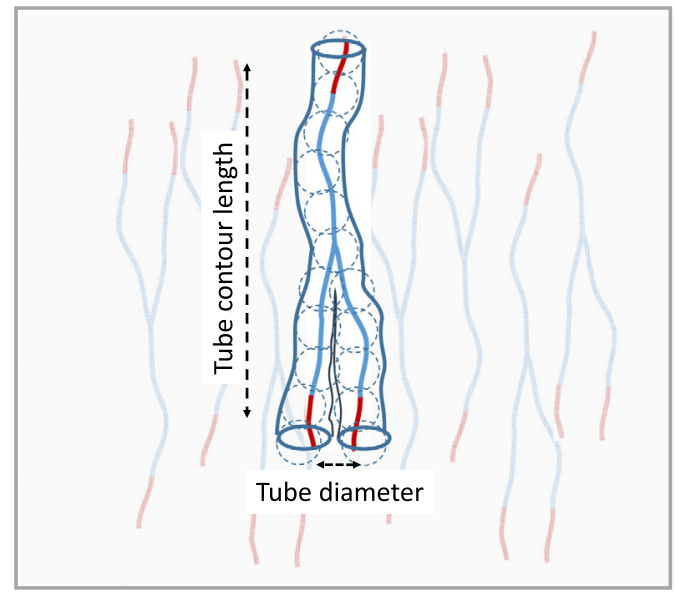

FIG. 5. Schematic figure of the stretched star block copolymer, illustrating the fully extended three-armed tube with two of the tube arms positioned right next to each other. center-to-center distance. While this may be fortuitous we suggest that the two arms do not constitute a common tube, but make up individual tubes separated with roughly two times their radius, thus giving rise to the pronounced correlation peak.

With the molar mass $\left(M_{w}=101.7 \mathrm{~kg} / \mathrm{mol}\right)$, the one-arm chain contour length is $R_{\max }(1 \mathrm{arm})=R_{\circ}^{2} / b=2480 \AA$, while the tube contour length is $L_{e}=Z a=520 \AA$. A fully stretched star polymer would have a total extension corresponding to two fully extended chains, i.e., $R_{\max }(\operatorname{star})=2 \times 2480 \AA=4960 \AA$. The total length of a fully extended tube of the star polymer is correspondingly given by the sum of the tube length of two arms, $2 L_{e}=2 Z a=1040 \AA$. This value is, with our experimental resolution, very close to the experimental value obtained from the vertical peaks, equal to $1300 \AA$, though indicating slight stretching. Our interpretation of the experimental scattering pattern is thus that of a simple three-armed tube that retains the tube characteristics, while being fully extended parallel to the flow, as sketched in Fig. 5.

Even though our proposed tube picture may be somewhat naive, it describes very well the properties of the experimental findings. We should admit that the tube model may not make much sense for a fully extended tube, since such a system no longer has entanglements. The studied polymer system has, however, been exposed to a stretching that is fast relative to typical relaxation mechanisms, and one may accept that the chains still have the memory of a virtual tube, within which they appear confined. Without the physical tube, however, it is not clear what will happen during relaxation from the extended configuration; whether the chain relaxation will still be dominated by a virtual tube, or at which stage a new tube can be defined.

A simple comparison would be that of a simple two-arm star-block copolymer, i.e., a linear tri-block PSd-PS-PSd polymer. Exposed to a similar mechanical strain, we would predict that the resulting SANS pattern would be restricted to the two "vertical" scattering streaks. We are planning such experiments.

In summary, we have shown that three-armed star molecules of similar block copolymer arms behave in accordance with the random phase approximation of Gaussian chains in the relaxed state. Upon exposure to large extensional flows, the polymer arms change conformation to a highly stretched structure that mimics that of a fully extended three-armed tube model, with one arm in one direction and the two other arms in the opposite direction on the flow axis. The two arms pointing in the same direction are parallel and their associated tubes are right next to each other.

This work was funded from the Independent Research Fund Denmark, Technology and Production (DFF-400500112), and the Danish Research Infrastructure via DANSCATT (SFU-7055-00005B). Q. H. would like to 
acknowledge financial support from the Aage and Johanne Louis Hansen Foundation. The Paul Scherrer Institute and ANSTO are acknowledged for neutron beam time at, respectively, SANS-I and Quokka.

*kell@nbi.dk

[1] P. G. de Gennes, J. Chem. Phys. 55, 572 (1971).

[2] M. Doi and S. F. Edwards, The Theory of Polymer Dynamics (Clarendon Press, Oxford, 1988).

[3] P. Schleger, B. Farago, C. Lartigue, A. Kollmar, and D. Richter, Phys. Rev. Lett. 81, 124 (1998).

[4] V. G. Sakai, C. Alba-Simionesco, and S.-H. Chen, Dynamics of Soft Matter, Neutron Applications (Springer, New York, 2012).

[5] D. J. Read and T. C. B. McLeish, Phys. Rev. Lett. 79, 87 (1997).

[6] A. E. Likhtman and T. C. B. McLeish, Macromolecules 35, 6332 (2002).

[7] W. Pyckhout-Hintzen, S. Westermann, A. Wischnewski, M. Monkenbusch, D. Richter, E. Straube, B. Farago, and P. Lindner, Phys. Rev. Lett. 110, 196002 (2013).

[8] Z. Wang et al., Phys. Rev. X 7, 031003 (2017).

[9] O. Hassager, K. Mortensen, A. Bach, K. Almdal, H. K. Rasmussen, and W. Pyckhout-Hintzen, Rheol. Acta 51, 385 (2012).

[10] J. J. K. Kirkensgaard, L. Hengeller, A. Dorokhin, Q. Huang, C. J. Garvey, K. Almdal, O. Hassager, and K. Mortensen, Phys. Rev. E 94, 020502(R) (2016).

[11] T. C. B. McLeish, Adv. Phys. 51, 1379 (2002).

[12] G. S. Grest, L. J. Fetters, J. S. Huang, and D. Richter, Star Polymers: Experiments, Theory, and Simulation, Advances in Chemical Physics Vol. 94, edited by I. Prigogine and S. A. Rice (John Wiley \& Sons, New York, 1996), ISBN 0471-14324-3.

[13] A. Dondos, D. Papanagopoulos, P. G. de Gennes, and F. Brochard-Wyart, Macromol. Theory Simul. 8, 147 (1999).

[14] X. Ye and T. Sridhar, Macromolecules 34, 8270 (2001).

[15] D. Vlassopoulos, G. Fytas, T. Pakula, and J. Roovers, J. Phys. Condens. Matter 13, R855 (2001).
[16] J. G. H. Cifre, R. Pamies, M. C. L. Martinez, and J. G. de la Torre, Polymer 46, 6756 (2005).

[17] M. Ripoll, R. G. Winkler, and G. Gompper, Phys. Rev. Lett. 96, 188302 (2006).

[18] N. H. Aloorkar, A. S. Kulkarni, R. A. Patil, and D. J. Ingale, Int. J. Pharm. Sci. Nanotechnol. 5, 1675 (2012).

[19] X. Xu and J. Chen, J. Chem. Phys. 144, 244905 (2016).

[20] Q. Huang, S. Agostini, L. Hengeller, M. Shivokhin, N. J. Alvarez, L. R. Hutchings, and O. Hassager, Macromolecules 49, 6694 (2016).

[21] G. Ianniruberto and G. Marrucci, Macromolecules 46, 267 (2013).

[22] A. Dorokhin, Entangled Polymer Melts in Extensional Flow. Synthesis, Rheology, Neutron Scattering, PhD Thesis, Technical University of Denmark, 2017.

[23] L. J. Fetters, D. J. Lohsey, and R. H. Colby, Physical Properties of Polymers Handbook, edited by J. E. Mark, 2nd ed. (Springer, New York, 2007), Vol. 3, pg. 25, ISBN 978-0-387-31235-4.

[24] L. Hengeller, Q. Huang, A. Dorokhin, K. Almdal, N. J. Alvarez, and O. Hassager, Rheol. Acta 55, 303 (2016).

[25] NIST Center for Neutron Research, Neutron Scattering Lengths (2018), https://www.ncnr.nist.gov/resources/nlengths/.

[26] L. Leibler, Macromolecules 13, 1602 (1980).

[27] M. Doi, Introduction to Polymer Physics (Clarendon Press, Oxford, 1996), ISBN 198517726.

[28] M. O. de la Cruz and I. C. Sanchez, Macromolecules 19, 2501 (1986).

[29] M. Guenza and A. Perico, Macromolecules 26, 4196 (1993).

[30] M. Watzlawek, H. Löwen, and C. Likos, J. Phys. Condens. Matter 10, 8189 (1998).

[31] A. Borger et al. (unpublished).

[32] M. Heinrich, W. Pyckhout-Hintzen, J. Allgaier, D. Richter, E. Straube, D. J. Read, T. C. B. McLeish, D. J. Groves, R. J. Blackwell, and A. Wiedenmann, Macromolecules 35, 6650 (2002).

[33] J. D. Londono, A. H. Narten, G. D. Wignall, K. G. Honnell, E. T. Hsieh, T. W. Johnson, and F. S. Bates, Macromolecules 27, 2864 (1994). 